\title{
Interactive Simulation of Rigid Body Interaction With Friction-Induced Sound Generation
}

\author{
Federico Avanzini, Stefania Serafin, and Davide Rocchesso
}

\begin{abstract}
Acoustic simulation of friction is a particularly challenging task, because continuous (strong) contact conditions require a tight and veridical integration of the synthesis layer with the control input. This paper presents an algorithmic realization that combines recently proposed physical models of friction with the lumped modal description of resonating bodies. It is shown that the resulting nonlinear dynamical system can be discretized using a numerical technique that allows efficient and accurate simulation. Applications in the context of interactive audio-visual animation on low-cost general-purpose computers are demonstrated, and an approach to joint audio-visual synthesis is proposed that provides fine-scale synchronization and high coherence between the two modalities. The interactive animations show that the model is successful in reproducing several salient everyday sound phenomena, such as rubbing, braking, and squeaky doors.
\end{abstract}

Index Terms-Audio-visual contact simulation, elasto-plastic friction models, modal synthesis, nonlinear acoustic systems, sound source modeling.

\section{INTRODUCTION}

$\mathbf{F}$ RICTION MODELING, as a branch of tribology, is a very active research area of applied mechanics, with applications in system control and robotics where the main objectives are friction prediction, elimination, or compensation. The topic of friction modeling is also addressed in the fields of computer graphics, haptics, and sound modeling, where the main objective is instead the simulation and rendering of sliding contact of rigid bodies. Due to strong nonlinear coupling, friction phenomena produce a wide variety of rich acoustic behaviors, even when very few resonating modes of the contacting objects are involved in the interaction. The temporal evolution of these sounds is capable to elicit our sense of effort very vividly, so that audition can even replace haptic functionalities in some applications.

Especially in interactive settings, such as games and virtual environments, sound and vision are inherently linked to each other. In order for the two modalities to provide mutual reinforcement and a coherent perceptual experience, audio-visual consistency must be achieved on a fine scale and continuously

Manuscript received November 6, 2003; revised August 16, 2004. This work was supported by the Sounding Object collaborative R\&D program under Contract IST-2000-25287. The Associate Editor coordinating the review of this manuscript and approving it for publication was Dr. Walter Kellerman.

F. Avanzini is with the Dipartimento di Ingegneria dell'Informazione, University of Padova, 35131 Padova, Italy (e-mail: avanzini@dei.unipd.it).

S. Serafin is with Copenhagen Institute of Technology, Aalborg University, DK-2750 Ballerup, Denmark (e-mail: sts@media.aau.dk).

D. Rocchesso is with the Dipartimento di Informatica, University of Verona, 37134 Verona, Italy (e-mail: rocchesso@sci.univr.it).

Digital Object Identifier 10.1109/TSA.2005.852984 in time. Simple techniques for audio-visual onset synchronization often lack to provide such coherence, especially in environments where events and processes are under direct user control. In this perspective, using physical models to generate both the low-rate large-scale phenomena (visual motion) and the highrate small-scale phenomena (sounds) is an attractive option.

In this paper we consider the complex mechanics of friction as a challenging testbed for integrated audio-visual physical modeling. First, a complete physical model is constructed, in which an advanced and versatile friction model [1] is used as the coupling mechanism between two modal resonators. Second, a robust and efficient technique is developed in order to discretize the model: we show that a recently proposed numerical method [2] can be extended to the problem here considered, and that the resulting algorithm runs easily in real-time on low-cost platforms. Finally, the model is applied to the simulation of a variety of relevant everyday friction phenomena. Audio-visual interactive animations are developed, in which fine scale audio-visual consistency is obtained by using the sound synthesis engine to drive both the graphics rendering and the audio feedback. All the modeled phenomena occur in strong-contact conditions and are nonlinearly and dynamically dependent on the imposed forces, thus being difficult to reproduce with prior-art audio-visual animation techniques.

Section II briefly reviews prior art in sound modeling for computer animation and in friction simulation. Section III describes the friction model of choice, while Section IV discusses its robust discretization. Section V details the model implementation, parametrization, and control. Finally, Section VI describes the interactive audio-visual simulations of everyday examples, namely a braking wheel, a squeaky door, and a rubbed wineglass.

\section{RELATED WORK}

\section{A. Sound Modeling for Computer Animation}

At present it is still common practice to add sound effects by hand onto film shots, even though automatic soundtrack production and synchronization for computer animations was proposed about a decade ago [3], [4]. In those works, prototype sounds, either recordings or synthesis models, are attached to objects and activated by sound event scripts. These scripts can be defined by the animator or automatically computed by physically-based motion control. Physically-based models offer a viable way to synthesize naturally behaving sounds from computational structures that can easily interact with the environment and respond to physical input parameters. In physically-based interactive systems, a gesture can produce 
consistent and natural feedback in terms of both visual motion and sound.

The physical behavior of interacting objects can be modeled with different approaches. Van den Doel et al. [5], [6] proposed modal synthesis [7] as an efficient yet accurate framework for describing the acoustic properties of objects. The parameters of modal resonators can be derived from geometric and physical descriptions of objects, or measured directly with scanning techniques [8]. The same synthesis technique has also been used by O'Brien et al. [9] in order to achieve real-time sound generation in interactive applications, where conventional rigidbody simulators are used. Their approach relies on finite element modeling of each object for precomputation of shapes and frequencies of the modes. At runtime, contact forces are used to drive the modal synthesizer, under the assumption that the sound-producing phenomena are linear, thus being representable as source-filter systems.

For noninteractive applications, it has been proposed to synthesize sound as a side effect of nonlinear finite element simulations [10]. In this way, sounds generated from complex nonlinear phenomena can be simulated, but the heavy computational load prevents the use of the method in interactive settings.

An approach to simulate complex dynamical systems as a multitude of interacting particles was proposed by Cadoz and coworkers [11], [12]. To achieve both visual and sonic animations, the CORDIS-ANIMA system was developed, in which discrete mass-spring-damper models are arranged in meshes where the interaction of these second-order systems can be locally made nonlinear. Again, large amounts of computations are required for large structures, but many relevant nonlinear phenomena can be mimicked.

Physical models are widely studied in the computer music community, and have been mainly applied to the faithful simulation of existing musical instruments. The use of physical modeling in conjunction with computer animation for the creation of a virtual orchestra is discussed in [13]. The techniques used in those simulations, however, lack of generality, since each physical system needs its own model. Cook has steered much of the expertise in musical sound synthesis toward the simulation of everyday sounds [14], especially in the context of interactive systems, where computational efficiency is a major concern. He coined the term physically-informed sonic modeling to refer to techniques which deviate from a direct fine-grained mathematical description of physical phenomena by employing statistical or signal-processing techniques. Extensive discussion is provided in [14] about sounds produced by sliding and scraping objects: friction modeling is presented as a challenging area for future research, because faithful, robust, versatile, and efficient models for bodies in strong-contact condition have not been proposed yet.

\section{B. Friction Modeling}

The word friction, traceable to 15th century English, denotes the force that resists the relative motion between two contacting bodies, and derives from the Latin word fricare, "to rub." Friction is a universal phenomenon, that appears in a mechanical system at the physical interface between two contacting surfaces
[15], [16]. It is not surprising therefore that the study of friction has a long history, which dates back to Aristotle and Leonardo.

A turning point toward realistic friction modeling were the observations made by Stribeck: by performing experiments on sliding bearings, he showed the dependence of the friction coefficient on the relative sliding velocity of two contacting bodies. Models that make use of a velocity-dependent friction coefficient are usually termed kinetic models (as the friction force is assumed to be a function of velocity only), or static models (since the force-velocity dependence is derived under stationary conditions). Kinetic models are typically used in the context of physically based animation, where friction forces need to be incorporated in the simulations of bodies in contact (see e.g., [17]). However, the detailed dependence of friction forces on velocity is usually not accounted for, nor is the internal dynamics of the friction mechanisms. Although it may be argued that these aspects are not relevant for animation, prior art in sound synthesis and automatic control have shown that they are crucial for audio applications and continuous control.

More recently, a class of dynamic friction models has been developed, where the dependence of friction on the relative sliding velocity is modeled using a differential equation. The starting point of the first dynamic model, proposed by Dahl [18], is the stress-strain curve of classic solid mechanics. This has been later modified and improved by the so-called LuGre (Lund-Grenoble) model [19], in which friction is described by a single-state system that represents the average plastic behavior of a multitude of micro-contacts. In order to avoid a nonphysical drift artifact that affects the LuGre model, a class of elasto-plastic models has been proposed [1]. This latter modeling approach is adopted in this paper, and the LuGre and elasto-plastic models are described in Section III-A.

Dynamic models are able to take into account presliding behavior, where the friction force increases gradually for small displacement values. Static and dynamic friction models have the same behavior at high or stationary relative velocities, but dynamic models provide more accurate simulation of transients [20], which is particularly relevant for realistic sound synthesis. Dynamic friction modeling has been also used for haptic applications: Hayward and Armstrong [21] describe an approach to haptic rendering based on an early formulation of the elastoplastic models. However, the literature of haptic computation is most often concerned with the simulation of surface roughness (see, e.g., [22] and [23]). In this context, stochastic texturing models are typically used, which are based on similar techniques developed in computer graphics.

Since different interacting dry surfaces are governed by similar friction mechanisms, it is possible to create a general framework for friction modeling. Moreover, given the nonlinear nature of the interaction, structures with few resonances are able to produce rich sonorities as the spectral content is largely determined by mode-locking effects: depending on the control exerted on the system, different normal modes-and their relative harmonic series-dominate the oscillation (see [24] for a general discussion on mode-locking in nonlinear acoustic systems). This is a relevant issue from a computational viewpoint, since efficient models can be developed that provide realistic simulations of contacting objects. When looking 


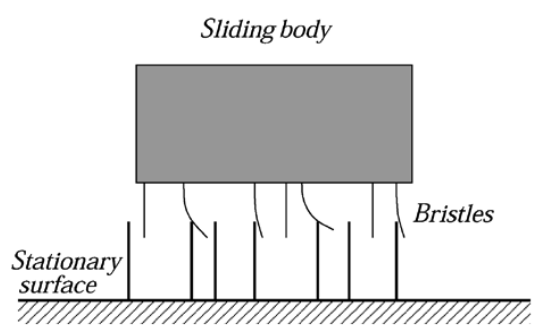

(a)

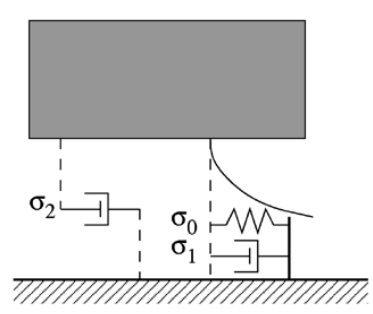

(b)

Fig. 1. (a) Bristle model and (b) the LuGre single-state averaged model.

for accurate reproduction of friction phenomena, however, it is necessary to bear in mind that "There are many different mechanisms. To construct a general friction model from physical first principles is simply not possible" [25].

\section{Physically-Based Friction Simulation}

The approach to simulation described in this section relies on the decomposition of a vibrating system into excitation and resonators. The resonating objects are modeled as lumped mechanical systems according to the modal synthesis approach [7], and are coupled through a nonlinear interaction mechanism.

\section{A. Friction Model}

The model adopted here is based on a "bristle-based" interpretation of frictional contact, as shown in Fig. 1(a).

Microscopic irregularities cause two facing surfaces to make contact at a number of asperities. This situation can be visualized as two rigid bodies that interact through a large number of elastic bristles. When a tangential force is applied, the bristles will randomly deflect like damped springs, each contributing a fraction of the total friction load. The load contributed by each bristle is assumed to be proportional to the strain of the bristle, i.e., the bristles act as linear springs. When the strain exceeds a certain level the bond is broken.

The LuGre friction model, developed by researchers at both Lund and Grenoble laboratories [19], captures and averages the random bristle behavior in a single-state system. Tangential forces affect the average bristle deflection and, if the deflection is large enough, the bristles start to slip. The LuGre model exhibits a spurious behavior, in that it always predicts drift when arbitrarily small forces or torques are applied. In order to overcome this problem, Dupont et al. [1] proposed a class of elasto-plastic models, described by the equations

$$
\begin{aligned}
\dot{z}(v, z) & =v\left[1-\alpha(v, z) \frac{z}{z_{\mathrm{ss}}(v)}\right] \\
f(z, \dot{z}, v, w) & =\sigma_{0} z+\sigma_{1} \dot{z}+\sigma_{2} v+\sigma_{3} w
\end{aligned}
$$

where $z$ is the average bristle deflection, $v$ is the relative velocity between the two surfaces, and $f$ is the resulting friction force. The coefficient $\sigma_{0}$ is the bristle stiffness, $\sigma_{1}$ is the bristle damping, and the term $\sigma_{2} v$ accounts for linear viscous friction [see Fig. 1(b)].

The function $\alpha(v, z)$ in (1) is an adhesion map that controls the rate of change of $z$. Parametrizations of $\alpha$ (see, e.g., (5) below) must guarantee minimal smoothness requirements, and must satisfy the properties $\alpha \equiv 0$ for $z$ small enough and $\alpha \equiv 1$ for $z$ large enough. More precisely, if the bristle displacement $z$ is smaller than a given breakaway displacement $z_{b a}$, then $\alpha \equiv 0$ and the model possesses a purely elastic presliding regime (that is, $\dot{z}=v$ from the first equation in (1)). Transition to the plastic regime occurs for large bristle displacements. The LuGre model is obtained as a special case of (1) with $\alpha(v, z) \equiv 1$ everywhere.

The function $z_{\mathrm{ss}}(v)$ that appears in (1) can be recognized to be the steady-state friction characteristic. From the first equation in (1), steady state conditions in the sliding regime (i.e., $\dot{z}=0$, with $v \neq 0, \alpha=1$ ) are met if and only if $z=z_{\mathrm{ss}}$.

A fourth component $\sigma_{3} w(t)$ has been added here to the second equation in (1), which is not part of the original formulation by Dupont et al. [1]. The term $w$ is related to surface roughness and is needed in order to simulate scraping and sliding effects, whereas the original elasto-plastic formulation [1] only accounts for stick-slip phenomena. Following [6], the $w$ component is modeled as fractal noise, i.e., noise with a power spectrum $W(\omega) \sim \omega^{\beta}$, where the exponent $\beta$ determines the fractal dimension of the noise signal and can in principle be used as a roughness-related parameter.

\section{B. Exciter-Resonator Model}

According to the theory of modal analysis [7], a vibrating object can be described by means of a set of normal modes of vibration, each associated with a specific frequency of oscillation and a specific spatial shape. The modes are decoupled and the modal displacements can be modeled as second order mechanical oscillators. Given a set of $N$ modes and a set of $N$ points on the vibrating object, the displacements at these points are obtained as a linear transformation of the modal displacements through a $N \times N$ modal shape matrix $T=\left[t_{l j}\right]$. While the modal description of continuous structures is an approximate one, modal frequencies and shapes can be derived analytically in the case of discrete structures (e.g., lumped masses coupled through springs and dampers).

In the remainder of the paper, one of the contacting modal objects is named "exciter" and the superscript $(e)$ is used when referring to its variables. Similarly, the "resonator" is indicated by the superscript $(r)$. These labels are merely conventional, and are really meaningful only when one object (the exciter) is much stiffer than the other (the resonator), e.g., as in bow-string interaction. Resonator and exciter are modeled as a set of $N^{(r)}$ and $N^{(e)}$ second order mechanical oscillators respectively, and they interact via the friction force given in (1). Assuming that 
the interaction occurs at a point $m=1 \ldots N^{(r)}$ of the resonator and point $l=1 \ldots N^{(e)}$ of the exciter, the equations are [26]

$$
\left\{\begin{array}{c}
\ddot{x}_{i}^{(e)}+g_{i}^{(e)} \dot{x}_{i}^{(e)}+\left[\omega_{i}^{(e)}\right]^{2} x_{i}^{(e)}=\frac{1}{m_{i}^{(e)}}\left(f_{e}^{(e)}+f\right) \\
\left(i=1 \ldots N^{(e)}\right) \\
\ddot{x}_{j}^{(r)}+g_{j}^{(r)} \dot{x}_{j}^{(r)}+\left[\omega_{j}^{(r)}\right]^{2} x_{j}^{(r)}=\frac{1}{m_{j}^{(r)}}\left(f_{e}^{(r)}-f\right) \\
\left(j=1 \ldots N^{(r)}\right) \\
v=v_{m, l}=\sum_{j=1}^{N^{(r)}} t_{m j}^{(r)} \dot{x}_{j}^{(r)}-\sum_{i=1}^{N^{(e)}} t_{l i}^{(e)} \dot{x}_{i}^{(e)}
\end{array}\right.
$$

where the $x$ variables represent the modal displacements, while the parameters $\omega^{(r)}, g^{(r)}$, and $m^{(r)}$ are the modal center frequencies, damping coefficients, and masses respectively. The terms $f_{e}^{(r)}$ and $f_{e}^{(e)}$ represent external forces, as indicated by the subscript $e$. The relative velocity $v$ at the interaction point is obtained from the modal velocities via the modal shape matrices $\left[t_{m j}^{(r)}\right],\left[t_{l i}^{(e)}\right]$. The complete system is therefore described by (1) and (2).

The steady-state friction characteristic $z_{\mathrm{sS}}$ is defined here as in [19]

$$
z_{\mathrm{sS}}(v)=\frac{\operatorname{sgn}(v)}{\sigma_{0}}\left[f_{c}+\left(f_{s}-f_{c}\right) e^{-\left(v / v_{s}\right)^{2}}\right]
$$

where $f_{c}, f_{s}$ are the Coulomb force and the stiction (short for static friction) force respectively, while $v_{s}$ is named Stribeck velocity. From the second equation in (1), the steady-state friction curve is

$$
f_{\mathrm{ss}}(v, w)=f\left(z_{\mathrm{ss}}(v), 0, v, w\right)=\sigma_{0} z_{\mathrm{ss}}(v)+\sigma_{2} v+\sigma_{3} w .
$$

Fig. 2 provides a plot of $f_{\mathrm{ss}}$. This has the typical shape used in static models of dry contact, and accounts for the so-called Stribeck effect, i.e., the decrease of the friction coefficient for increasing relative sliding velocity.

Following Dupont et al. [1], we parametrize the function $\alpha(v, z)$ as

$$
\begin{aligned}
& \alpha(v, z) \\
& \quad= \begin{cases}0, & |z|<z_{b a}, \quad \operatorname{sgn}(v)=\operatorname{sgn}(z) \\
\alpha_{m}(v, z), & z_{b a}<|z|<z_{\mathrm{ss}}(v), \quad \operatorname{sgn}(v)=\operatorname{sgn}(z) \\
1, & |z|>z_{\mathrm{ss}}(v), \quad \operatorname{sgn}(v)=\operatorname{sgn}(z) \\
0, & \operatorname{sgn}(v) \neq \operatorname{sgn}(z)\end{cases}
\end{aligned}
$$

where $z_{b a}$ is the breakaway displacement below which the presliding is purely elastic, and

$$
\alpha_{m}(v, z)=\frac{1}{2}\left[1+\sin \left(\pi \frac{z-\frac{1}{2}\left(z_{\mathrm{ss}}(v)+z_{b a}\right)}{z_{\mathrm{ss}}(v)-z_{b a}}\right)\right]
$$

ensures a smooth transition between elastic and plastic behavior. The equations given in (3) and (5) are just two possible parametrizations for the functions $z_{\mathrm{ss}}(v)$ and $\alpha(v, z)$. In practice, different parametrizations can be used without affecting the model behavior significantly.

\section{DisCRETE-Time Equations}

This section addresses the computational issues involved in the discretization of the differential system (1), (2). It is known [2] that delay-free paths can be generated in the discrete-time

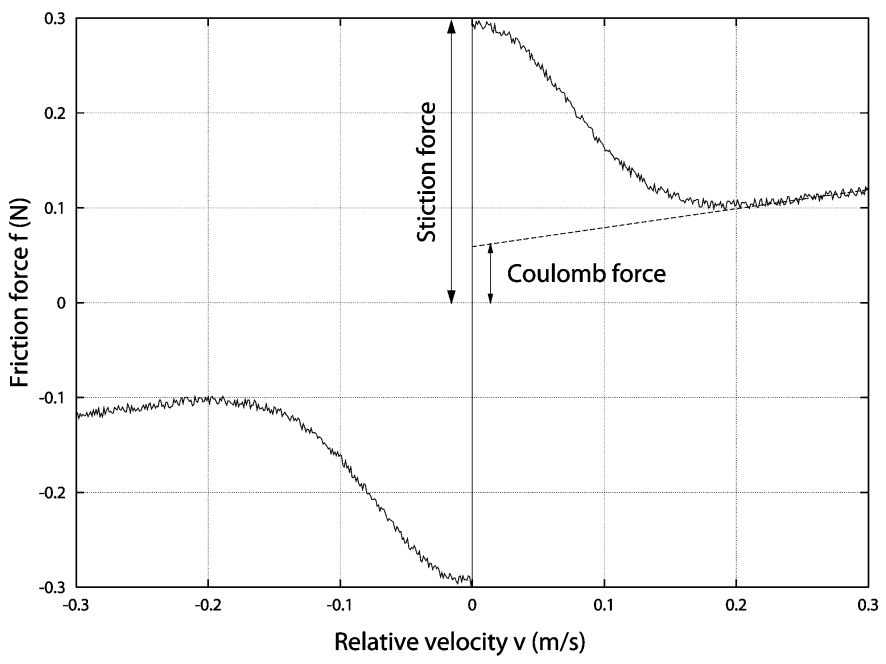

Fig. 2. Steady-state friction force. Solid line: $f_{\mathrm{ss}}(v, w)$. Dotted line: asymptotic behavior $f_{c}+\sigma_{2} v$.

system, so that implicit nonlinear relations must be solved. A general procedure, the so-called $\mathrm{K}$ method, has been proposed in [2], that provides an efficient and accurate solution to such numerical problems.

\section{A. System Decomposition}

In order to show that system (1), (2) can be dealt with using the $\mathrm{K}$ method, we show that it fulfills the main hypothesis assumed in [2], i.e., it can be written in the general form

$$
\left\{\begin{array}{l}
\dot{\boldsymbol{w}}=\boldsymbol{A w}+\boldsymbol{B u}+\boldsymbol{C y}, \\
\boldsymbol{y}=\boldsymbol{f}_{\mathbf{N L}}(\boldsymbol{x}), \quad \text { with } \boldsymbol{x}=\boldsymbol{D} w
\end{array}\right.
$$

in which a linear filter contains all the system dynamics and is coupled to a nonlinear map $f_{\mathbf{N L}}$. Note that the system under consideration here is not trivially represented with this structure, since the interaction is described through a nonlinear differential equation (1) rather than an instantaneous nonlinear map. However, the decomposition (7) can still be used if a global state vector $\boldsymbol{w}$ is constructed, which incorporates the bristle state $z$.

Assume without loss of generality that $N^{(r)}=N^{(e)}=1$. Then the system is in form (7) with the definitions

$$
\boldsymbol{w}=\left[\begin{array}{c}
x^{(r)} \\
v^{(r)} \\
x^{(e)} \\
v^{(e)} \\
z
\end{array}\right], \quad \boldsymbol{u}=\left[\begin{array}{c}
f_{e}^{(r)} \\
f_{e}^{(e)} \\
w
\end{array}\right], \quad \boldsymbol{x}=\left[\begin{array}{c}
v \\
z
\end{array}\right], \quad y=\dot{z}
$$

$$
\boldsymbol{A}=-1
$$

$$
\cdot\left[\begin{array}{ccccc}
0 & 1 & 0 & 0 & 0 \\
\omega^{(r) 2} & g^{(r)}+\frac{\sigma_{2} t_{1}^{(r)}}{m^{(r)}} & 0 & \frac{-\sigma_{2} t_{1}^{(e)}}{m^{(r)}} & \frac{\sigma_{0}}{m^{(r)}} \\
0 & 0 & 0 & -1 & 0 \\
0 & \frac{-\sigma_{2} t_{1}^{(r)}}{m^{(e)}} & \omega^{(e) 2} & g^{(e)}+\frac{\sigma_{2} t_{1}^{(e)}}{m^{(e)}} & \frac{-\sigma_{0}}{m^{(e)}} \\
0 & 0 & 0 & 0 & 0
\end{array}\right] \text {, }
$$

$\boldsymbol{B}=\left[\begin{array}{ccc}0 & 0 & 0 \\ \frac{1}{m^{(r)}} & 0 & -\frac{\sigma_{3}}{m^{(r)}} \\ 0 & 0 & 0 \\ 0 & \frac{1}{m^{(e)}} & \frac{\sigma_{3}}{m^{(e)}} \\ 0 & 0 & 0\end{array}\right], \quad \boldsymbol{C}=\left[\begin{array}{c}0 \\ -\frac{\sigma_{1}}{m^{(r)}} \\ 0 \\ \frac{\sigma_{1}}{m^{(e)}} \\ 1\end{array}\right]$,

$\boldsymbol{D}=\left[\begin{array}{ccccc}0 & t_{1}^{(r)} & 0 & -t_{1}^{(e)} & 0 \\ 0 & 0 & 0 & 0 & 1\end{array}\right]$ 
and with $f_{\mathrm{NL}}(v, z)=v\left[1-\alpha(v, z)\left(z / z_{\mathrm{ss}}(v)\right)\right]$. Generalization to the case $N^{(r)}>1, N^{(e)}>1$ is straightforward. Note that the values of $N^{(r)}, N^{(e)}$ only affect the dimension of the state vector $\boldsymbol{w}$, but not that of the input $\boldsymbol{x}$ to the nonlinear map. Therefore $f_{\mathrm{NL}}: \mathbb{R}^{2} \rightarrow \mathbb{R}$ regardless of the number of resonances.

System (7) provides a continuous-time state-space representation for the coupled modal resonators, which is discretized using the bilinear transformation. This discretization technique provides a reasonable trade-off between stability/accuracy properties and efficiency of the discrete-time system: its order of accuracy can be seen [27] to be two; it is an unconditionally stable method (since the left-half $s$-plane is mapped into the unit $z$-circle); it preserves the order of the continuous-time system.

One can verify that, when the system is discretized using the bilinear transformation [28], the vector $\boldsymbol{x}$ at time $n$ is written as $\boldsymbol{x}(n)=\tilde{\boldsymbol{x}}(n)+\boldsymbol{K} y(n)$, where the vector $\tilde{\boldsymbol{x}}(n)$ depends only on the inputs $\boldsymbol{u}$ and past values of $\boldsymbol{w}$ and $y$, and is therefore computable at time $n$. In fact one can verify that this result holds for any linear implicit numerical method (e.g., the family of multistep Adams-Moulton methods [27] which comprises the bilinear transformation). As a consequence the nonlinear map is rewritten in the discrete-time domain as

$$
y(n)=f_{\mathrm{NL}}(\tilde{\boldsymbol{x}}(n)+\boldsymbol{K} y(n)) .
$$

If the bilinear transformation is used, the matrix $K$ is found as $\boldsymbol{K}=\boldsymbol{D}\left(2 F_{s} \cdot \boldsymbol{I}-\boldsymbol{A}\right) \boldsymbol{C}$, where $F_{s}$ is the sampling frequency.

Equation (10) shows that a delay-free path is generated in the computation, as $y(n)$ is defined implicitly as a function of $\tilde{\boldsymbol{x}}(n)$. It is shown in [2] that a solution can be found either by precomputing the function $y(\tilde{\boldsymbol{x}})$ and storing it in a look-up table, or by using Newton-Raphson iterations to solve (10). In the latter case, which is more efficiently handled in case of multivariable nonlinear functions such as (10), the value of $y(n)$ is computed at each time step $n$ by finding a local zero of the function $g(y)=\left[f_{\mathrm{NL}}(\tilde{\boldsymbol{x}}+\boldsymbol{K} y)-y\right]$.

\section{B. System Equations}

After application of the bilinear transformation to system (7), the two resonating objects appear in the discrete-time domain as two banks of second-order bandpass filters, each one accounting for one specific mode of the objects. The resonator is represented by the discrete-time equations

$$
\begin{cases}\boldsymbol{x}_{j}^{(r)}(n)=A_{j}^{(r)} \boldsymbol{x}_{j}^{(r)}(n-1)+\boldsymbol{b}_{j}^{(r)}\left[f^{(r)}(n)+f^{(r)}(n-1)\right], & \left(\text { for } j=1 \ldots N^{(r)}\right), \\ f^{(r)}(n)=f_{e}^{(r)}(n)-f(n) & \end{cases}
$$

where the vectors $\boldsymbol{x}_{j}^{(r)}$ are defined as $\boldsymbol{x}_{j}^{(r)}=\left[x_{j}^{(r)}, \dot{x}_{j}^{(r)}\right]^{T}$, and $f^{(r)}$ is the total force acting on the resonator. The matrices are given by

$$
\begin{aligned}
\boldsymbol{A}_{j}^{(r)} & =\frac{1}{\Delta_{j}^{(r)}}\left[\begin{array}{cc}
\Delta_{j}^{(r)}-\omega_{j}^{(r) 2} / 2 & F_{s} \\
-F_{s} \omega_{j}^{(r) 2} & 2 F_{s}^{2}-\Delta_{j}^{(r)}
\end{array}\right] \\
\boldsymbol{b}_{j}^{(r)} & =\frac{1}{m_{j}^{(r)}} \cdot \frac{1}{4 \Delta_{j}^{(r)}}\left[\begin{array}{c}
1 \\
2 F_{s}
\end{array}\right]
\end{aligned}
$$

where $F_{s}$ is the sampling frequency and the quantity $\Delta_{j}^{(r)}$ is given by $\Delta_{j}^{(r)}=F_{s}^{2}+g_{j}^{(r)} F_{s} / 2+\omega_{j}^{(r) 2} / 4$. Identical equations, with matrices $A_{j}^{(e)}$ and vectors $\boldsymbol{b}_{j}^{(e)}$ (for $j=1 \ldots N^{(e)}$ ) are found for the exciter.

The last element of the state vector $\boldsymbol{w}$ (i.e., the bristle state $z$ ) is described in the discrete-time domain as

$$
\begin{aligned}
z(n) & \approx \underbrace{z(n-1)+\frac{1}{2 F_{s}} y(n-1)}_{\tilde{z}(n)}+\underbrace{\frac{1}{2 F_{s}}} y(n) \\
& =\boldsymbol{K}_{(2)} y(n)
\end{aligned}
$$

which realizes the decomposition $\boldsymbol{x}(n)=\tilde{\boldsymbol{x}}(n)+\boldsymbol{K} y(n)$ for the component $[\boldsymbol{x}]_{2}$.

The relative velocity $v(n)$ can also be found as the sum of its computable part $\tilde{v}(n)$ and the instantaneous contribution of $y(n)$. Recalling the definition of $v$ in (2), and exploiting the relations given in (11) and (13), $v(n)$ can be written as

$$
\begin{aligned}
v(n) & =\tilde{v}(n)+\boldsymbol{K}(1) y(n), \quad \text { with } \\
K(1) & =-\frac{b}{1+\sigma_{2} b}\left(\frac{\sigma_{0}}{2 F_{s}}+\sigma_{1}\right)
\end{aligned}
$$

where $b=\left[\sum_{i=1}^{N^{(e)}} t_{l i}^{(e)} \boldsymbol{b}_{i}^{(e)}(2)+\sum_{j=1}^{N^{(r)}} t_{m j}^{(r)} b_{j}^{(r)}(2)\right]$.

In conclusion, application of the bilinear transformation results in a discrete-time nonlinear map of the form (10), to which the $\mathrm{K}$ method is applied. Analysis on the simulations in a large region of the parameter space has shown that at most seven iterations of the Newton-Raphson algorithm typically allow convergence to the solution of (10). The iterative zero-finding procedure is therefore computationally efficient for real-time implementation.

\section{IMPLEMENTATION AND CONTROL}

The friction model described in Section III has been implemented as a plugin to pd (Pure Data'), an open source real-time synthesis environment developed by Miller Puckette and widely used in the computer music community. The model is part of a wider physically-based sound synthesis package, which implements a variety of interactions between modal resonators. ${ }^{2}$

The package is organized in an object oriented structure and includes the following subdirectories:

- Resonators: data structures and methods of modal resonators are defined. External forces can be applied at specified interaction points, each point being described by a set of numbers that weigh each mode at that point. Displacement or velocity are returned as outputs from the modal object.

- Interactors: for friction interaction, a function computes the forces to be applied to two interacting resonators according to the numerical scheme described in Section IV.

- Sound_modules: the final sound synthesis modules are obtained by specifying topologies in which resonators and interactors are combined (e.g., two resonators linked through a friction interactor, as in Fig. 3), and the external appearance (default parameter values, inlets and outlets) of the modules are defined.

1http://crca.ucsd.edu/ msp/

${ }^{2}$ The plugins are freely available, see the web site of the Sounding Object EU project, http://www.soundobject.org 


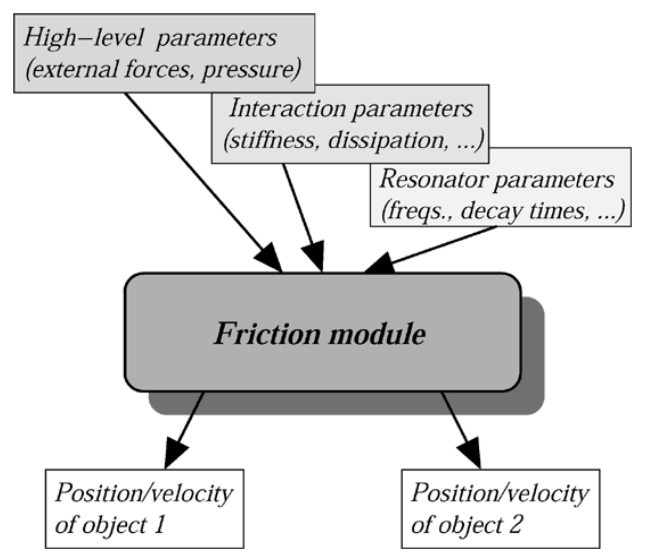

Fig. 3. Schematic representation of the friction model implementation.

The remainder of this section provides a physical and phenomenological description of the control parameters listed in Fig. 3.

High-level interactions between the user and the audio objects rely mainly upon three parameters. These are the external forces $f_{e}^{(e)}$ and $f_{e}^{(r)}$ defined in (2), that act on each of the two objects tangentially to the sliding direction, and the normal force $f_{N}$ between the two objects. It must be noted that treating $f_{N}$ as a control parameter is a simplifying assumption, since oscillatory normal force components always accompany the friction force in real systems [16]. The remaining parameters belong to a lower level control layer, as they are less likely to be touched by the user and have to be tuned at the sound design level.

Such low-level parameters can be grouped into two subsets, depending on whether they are related to the resonators' internal properties or to the interaction mechanism. Each mode of the two resonating objects is tuned according to its center frequency and decay time. It has been shown [29] that these parameters are strictly related to the perception of material. Additionally, a modal gain (which is inversely proportional to the modal mass) can be set for each resonator mode, and controls the extent to which the mode can be excited during the interaction. The implementation allows position dependent interaction by giving the option to choose any number of interaction points. A different set of modal gains can be set for each point.

A second subset of low-level parameters relates to the interaction force specification. The triple $\left(\sigma_{0}, \sigma_{1}, \sigma_{2}\right)$ defined in (1) assigns values to the bristle stiffness, the bristle internal dissipation, and the viscous friction, and therefore affects the characteristics of signal transients as well as the ease in establishing stick-slip motion. The triple $\left(f_{c}, f_{s}, v_{s}\right)$ defined in (3) specifies the shape of the steady state force. Specifically, the Coulomb force and the stiction force are related to the normal force through the equations $f_{s}=\mu_{s} f_{N}$ and $f_{c}=\mu_{d} f_{N}$, where $\mu_{s}$ and $\mu_{d}$ are the static and dynamic friction coefficients. Finally, the breakaway displacement $z_{b a}$ introduced in (5) is also influenced by the normal force. In order for the function $\alpha(v, z)$ to be well defined, the inequality $z_{b a}<z_{\mathrm{ss}}(v) \forall v$ must hold. Since $\min _{v} z_{\mathrm{ss}}(v)=f_{c} / \sigma_{0}$, a suitable mapping between $f_{N}$ and $z_{b a}$ is

$$
z_{b a}=c f_{c} / \sigma_{0}=c \mu_{d} f_{N} / \sigma_{0}, \quad \text { with } c<1 .
$$

One approach to determine the low-level model parameters is "hand and hear" direct manipulation. Since many "knobs" are
TABLE I

A Phenomenological Guide to Model Variables

\begin{tabular}{l|l|l}
\hline Symbol & Physical Description & $\begin{array}{l}\text { Phenomenological } \\
\text { Description }\end{array}$ \\
\hline$\sigma_{0}$ & bristle stiffness & $\begin{array}{l}\text { affects the evolution of } \\
\text { mode locking }\end{array}$ \\
\hline$\sigma_{1}$ & bristle damping & $\begin{array}{l}\text { affects the sound band- } \\
\text { width }\end{array}$ \\
\hline$\sigma_{2}$ & viscous friction & $\begin{array}{l}\text { affects the speed of } \\
\text { timbre evolution and } \\
\text { the sound pitch }\end{array}$ \\
\hline$\sigma_{d}$ & noise coefficient & $\begin{array}{l}\text { affects the perceived } \\
\text { surface roughness }\end{array}$ \\
\hline$\mu_{s}$ & static friction coeff. & $\begin{array}{l}\text { high values reduce the } \\
\text { sound bandwidth }\end{array}$ \\
\hline$v_{s}$ & Stribeck velocity & $\begin{array}{l}\text { affects the smoothness } \\
\text { of sound attack } \\
\text { of sound attack }\end{array}$ \\
\hline$f_{N}$ & normal force & $\begin{array}{l}\text { high values give } \\
\text { rougher and louder } \\
\text { sounds }\end{array}$ \\
\hline
\end{tabular}

available, the phenomenological description of model parameters given in Table I can be a helpful starting point for the sound designer. Besides direct empirical search, modal parameters can in certain cases be found in closed form (only for simple geometries), can be obtained from analysis of recorded sounds of real objects [8], or derived from finite element object modeling [10]. Interaction parameters can also be found from analysis of real signals. Parameter estimation techniques are the subject of many studies in automatic control, an extensive discussion of such issues is provided in [20]. In certain cases typical parameter values can be found from the literature (see, e.g., [30] for bowed string instruments).

\section{Audio-Visual Simulations}

The friction model presented in Sections III to V has been applied to several examples of acoustic systems with frictional induced vibrations. In order to test the potential applications of the model in interactive multimodal environments, simple animations have been generated for each example, ${ }^{3}$ which share a common scheme for the audio-visual rendering.

- The user controls one of the virtual objects in the scene. A standard pointing device is used for the control input, and the $(x, y)$ coordinates of the pointer are linearly mapped to the external force $f_{e}^{(e)}$ acting on the exciter and the normal force $f_{N}$, respectively.

- The synthesis engine returns positions and velocities of the interacting objects, at audio sample rate. These signals are used to drive both the graphics renderer and the audio feedback.

This approach allows for a high degree of interactivity. Moreover, it demonstrates that a single physical synthesis engine can be used for both graphics and audio. One main consequence is that the two modalities are highly consistent and synchronized on a fine scale.

${ }^{3}$ Video shots of the interactive animations can be downloaded. See the web site of the Sounding Object EU project, http://www.soundobject.org 


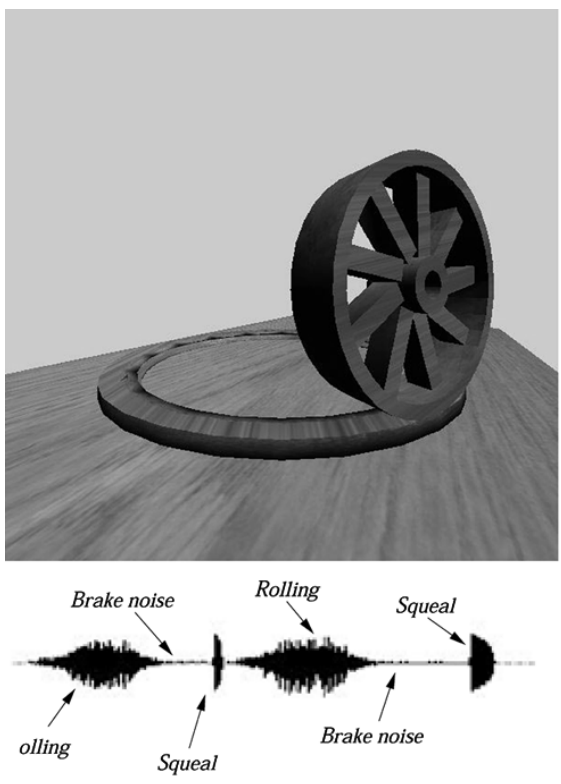

(a)
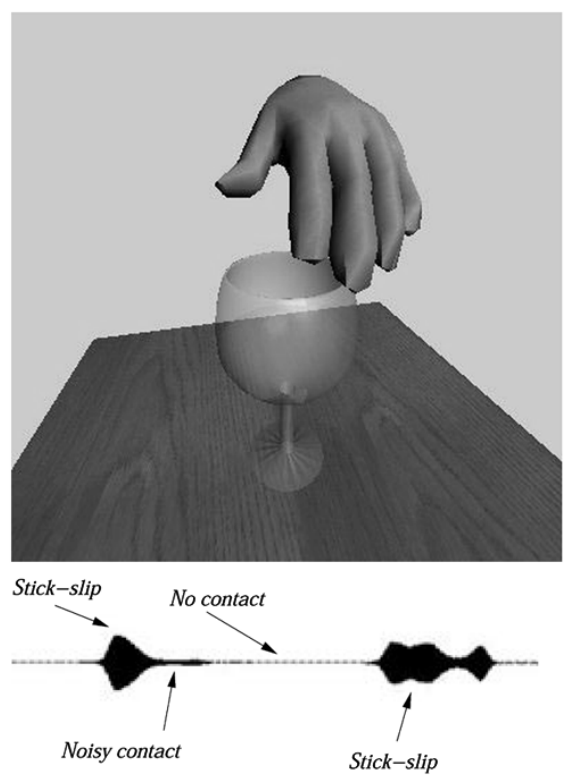

(b)

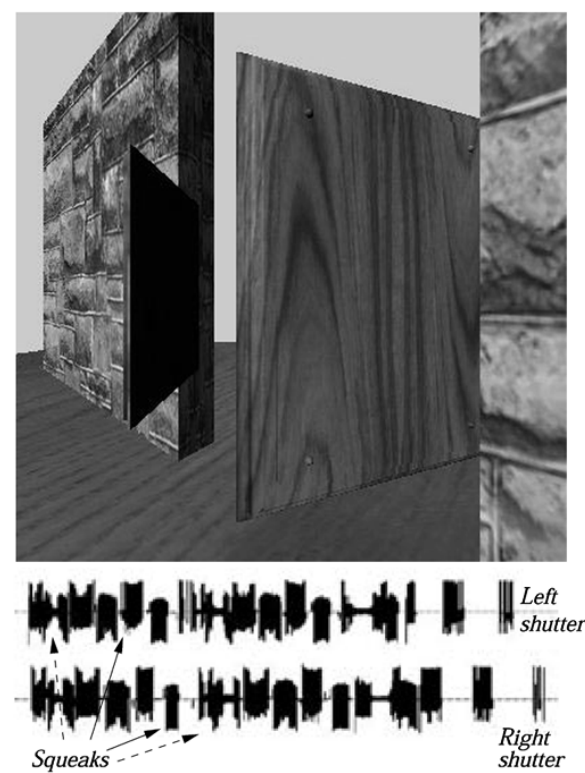

(c)

Fig. 4. Three-dimensional animations and waveforms: (a) a wheel which rolls, slides and eventually squeaks on a circular track; (b) a moist finger rubbing a crystal glass; and (c) a swinging door, each of the two shutters is linked to a friction module.

The animations represent examples of everyday friction sounds, and have been designed using external graphical libraries of $\mathrm{pd}$, specifically the OpenGL-based gem. ${ }^{4}$ In all the examples the main sound generation mechanism is the stick-slip relative motion.

Braking Effects: Different kinds of vibrations and sonorities develop within brakes, through several mechanisms. In the case of rotating wheels that slip sideways across the rails, the friction forces acting at the wheel rim excite transverse vibrations. In order to test the ability of the model to simulate brake noise, the simulation depicted in Fig. 4(a) has been designed, where a wheel driven by the external force $f_{e}^{(e)}$ rolls on a circular track. The rolling sound is obtained as the result of a sequence of micro-impacts (a detailed description of rolling sound design is out of the scope of this paper; see [31] for details). When a positive normal force is applied, the wheel is blocked from rolling and the friction model is triggered. Neat stick-slip is established only at sufficiently low velocities, and brake squeals are produced in the final stage of deceleration. The resulting effect convincingly mimics real brake noise.

Wineglass Rubbing: An excitation mechanism analogous to wheel-brake interaction appears when a wineglass is rubbed around its rim with a moist finger. In this case sound is generated at one of the natural frequencies of the glass [32] and its harmonics. Glasses with low damped resonances, such as crystal wineglasses, when rubbed produce melodies that have for a long time entertained guests around dinner tables.

By properly adjusting the modal frequencies and the decay times of the modal object that acts as the resonator, a distinctive glassy character can be obtained. The example depicted in Fig. 4(b) uses a resonator with two modes, where the parameter values have been obtained from Fourier analysis of a recorded sound from a real crystal glass. As in the previous example, the rubbing finger is controlled through mouse input. Interestingly,

${ }^{4}$ http://gem.iem.at/ setting the glass into resonance is not a trivial task and requires some practice and careful control, just as in the real world.

Door Squeaks: The third everyday sound which has been simulated is the squeak produced by the hinges of a swinging door. In this situation, different combinations of transient and continuous sliding produce many squeaks which create a broad range of sonic responses. The example depicted in Fig. 4(c) uses two exciter-resonator pairs, one for each of the shutters. In this case the modal frequencies of the objects have been chosen by hand-and-hear tuning on the basis of recorded sounds. The results are especially convincing in reproducing complex transient and glissando effects which are typically found in real door squeaks.

\section{CONCLUSION}

In this paper, we have described a physically based approach to audio rendering of continuous contact between rubbed surfaces. As far as sound modeling is concerned, the contributions of the paper can be summarized as follows: the proposed algorithm combines modal synthesis with a single-state nonlinear friction model that accurately reproduce stick-slip interactions; a pseudo-random component has been included in the definition of the friction force, thus allowing to simulate a wider class of frictional interactions including scraping and sliding; the numerical implementation of the model is obtained through a system decomposition into a linear differential system coupled to a memoryless nonlinear map. Due to this decomposition, existing accurate and efficient numerical methods can be adapted to the proposed model. Sound modules that implement the algorithm demonstrate that it allows real-time audio synthesis in interactive settings.

An approach to audio-visual interactive simulations has been proposed, in which the physical output from the synthesis engine is exploited to drive the rendering of both modalities. It has been pointed out that audio-visual coherence can greatly 
benefit from this approach. The proposed applications have shown that the physical description provided by the model is flexible enough to simulate a variety of complex everyday sounds which are generated by frictional interactions. Other possible applications include the simulation of bowed string musical instruments: a first study in this direction is documented in [33].

The applications also demonstrate the high degree of interactivity of the proposed sound model. Again, the accurate physical description of the interaction layer (i.e., the friction mechanisms) enables the user to experience realistic acoustic feedback from the virtual objects and enhance the sense of immersion in interactive simulations.

Other authors have stressed the importance of linear modal synthesis with position-dependent precomputed excitation as a tool to re-create complex sounds [6]. Our results suggest that, in the case of strong contact conditions, more refined models of the excitation are needed. The accurate modeling of the nonlinear friction mechanisms is at least as important in determining the sound quality as the detailed modal description of the resonating objects: no more than two modes for each resonator have been used in all the applications, and the spectral content of the audio signals is largely determined by mode-locking effects.

\section{ACKNOWLEDGMENT}

The authors thank M. Rath for his contribution to pd plugin design and implementation.

\section{REFERENCES}

[1] P. Dupont, V. Hayward, B. Armstrong, and F. Altpeter, "Single state elasto-plastic friction models," IEEE Trans. Automat. Contr., vol. 47, no. 5, pp. 787-792, 2002.

[2] G. Borin, G. De Poli, and D. Rocchesso, "Elimination of delay-free loops in discrete-time models of nonlinear acoustic systems," IEEE Trans. Speech Audio Process., vol. 8, no. 5, pp. 597-606, 2000.

[3] T. Takala and J. Hahn, "Sound rendering," in Proc. ACM SIGGRAPH 1993, J. Kajiya, Ed., Anaheim, CA, 1993, pp. 211-220.

[4] J. Hahn, J. Geigel, J. Lee, L. Gritz, T. Takala, and S. Mishra, "An integrated approach to audio and motion," J. Vis. Comp. Animat., vol. 6, no. 2, pp. 109-129, 1995.

[5] K. van den Doel and D. Pai, "Synthesis of shape dependent sounds with physical modeling," in Proc. Int. Conf. on Auditory Display (ICAD96), Palo Alto, CA, 1996.

[6] K. van den Doel, P. Kry, and D. Pai, "Foleyautomatic: Physically-based sound effects for interactive simulation and animation," in Proc. ACM SIGGRAPH 2001, E. Fiume, Ed., Los Angeles, CA, 2001, pp. 537-544.

[7] J. M. Adrien, "The missing link: Modal synthesis," in Representations of Musical Signals, G. De Poli, A. Piccialli, and C. Roads, Eds. Cambridge, MA: MIT Press, 1991, pp. 269-297.

[8] D. Pai, K. van den Doel, D. L. James, J. Lang, J. E. Lloyd, J. L. Richmond, and S. H. Yau, "Scanning physical interaction behavior of 3D objects," in Proc. ACM SIGGRAPH 2001, E. Fiume, Ed., Los Angeles, CA, 2001, pp. 87-96.

[9] J. O'Brien, C. Shen, and C. Gatchalian, "Synthesizing sounds from rigidbody simulations," in Proc. ACM SIGGRAPH 2002 Symp. Comp. Animation, San Antonio, TX, 2002, pp. 175-182.

[10] J. O'Brien, P. R. Cook, and G. Essl, "Synthesizing sounds from physically based motion," in Proc. ACM SIGGRAPH 2001, E. Fiume, Ed., Los Angeles, CA, 2001, pp. 529-536.

[11] C. Cadoz, A. Luciani, and J.-L. Florens, "Physical models for music and animated image. The use of CORDIS-ANIMA in Esquisses: A Music film by Acroe," in Proc. Int. Computer Music Conf. (ICMC'94), Aarhus, Denmark, 1994.

[12] J. Nouiri, C. Cadoz, and A. Luciani, "The physical modeling of complex physical structures. the mechanical clockwork: Motion, image, and sound," in Proc. IEEE Conf. Comp. Animation (CA'96), Geneva, Switzerland, 1996, pp. 36-46.
[13] L. Savioja, J. Huopaniemi, T. Lokki, and R. Väänänen, "Virtual environment simulation-Advances in the DIVA project," in Proc. Int. Conf. on Auditory Display (ICAD97), Palo Alto, CA, 1997, pp. 43-46.

[14] P. R. Cook, Real Sound Synthesis for Interactive Applications. Natick, MA: Peters, 2002.

[15] F. Elmer, "Nonlinear dynamics of dry friction.," J.Phys. A: Math. Gen., vol. 30, pp. 6057-6063, 1997.

[16] A. Akay, "Acoustics of friction," J. Acoust. Soc. Amer, vol. 111, no. 4, pp. $1525-1548,2002$.

[17] D. Baraff, "Fast contact force computation for nonpenetrating rigid bodies," in Proc. ACM SIGGRAPH 1994, A. Glassner, Ed., Orlando, FL, 1994, pp. 23-34.

[18] P. Dahl, "A Solid Friction Model," The Aerospace Corporation, El Segundo, CA, Tech. Rep. TOR-158, 1968.

[19] C. C. de Wit, H. Olsson, K. J. Åström, and P. Lischinsky, "A new model for control of systems with friction," IEEE Trans. Automat. Contr., vol. 40, no. 3, pp. 419-425, 1995.

[20] F. Altpeter, "Friction Modeling, Identification and Compensation," Ph.D. dissertation, École Polytechnique Fédérale de Lausanne, Lausanne, Switzerland, 1999.

[21] V. Hayward and B. Armstrong, "A new computational model of friction applied to haptic rendering," in Experimental Robotics VI, P. Corke and J. Trevelyan, Eds. New York: Springer-Verlag, 2000, pp. 403-412.

[22] J. Fritz and K. Barner, "Stochastic models for haptic texture," in Proc. SPIE Int. Symp. on Intelligent Systems and Advanced Manufacturing-Telemanipulator and Telepresence Technologies III, Boston, MA, 1996.

[23] J. Siira and D. K. Pai, "Haptic texturing-A stochastic approach," in Proc. IEEE Conf. on Robotics and Automation, Minneapolis, MN, 1996, pp. $557-562$.

[24] N. H. Fletcher, "Mode locking in nonlinearly excited inharmonic musical oscillators," J. Acoust. Soc. Amer, vol. 64, no. 6, pp. 1566-1569, Dec. 1978.

[25] H. Olsson, K. J. Åström, C. C. de Wit, M. Gäfwert, and P. Lischinsky, "Friction models and friction compensation," Eur. J. Contr., vol. 4, no. 3, pp. 176-195, 1998.

[26] F. Avanzini, S. Serafin, and D. Rocchesso, "Modeling interactions between rubbed dry surfaces using an elasto-plastic friction model," in Proc. COST-G6 Conf. on Digital Audio Effects (DAFx-02), Hamburg, Germany, Sept. 2002, pp. 111-116.

[27] J. D. Lambert, Numerical Methods for Ordinary Differential Systems. New York: Wiley, 1993.

[28] A. V. Oppenheim and R. W. Schafer, Discrete-Time Signal Processing. Englewood Cliffs, NJ: Prentice-Hall, 1989.

[29] R. L. Klatzky, D. K. Pai, and E. P. Krotkov, "Perception of material from contact sounds," Presence, vol. 9, no. 4, pp. 399-410, 2000.

[30] A. Askenfelt, "Measurement of the bowing parameters in violin playing. II: Bow-bridge distance, dynamic range, and limits of bow force," $J$. Acoust. Soc. Amer., vol. 86, no. 2, pp. 503-516, 1989.

[31] M. Rath, "An expressive real-time sound model of rolling," in Proc. COST-G6 Conf. on Digital Audio Effects (DAFx-03), London, U.K., 2003, pp. 165-168.

[32] T. D. Rossing, "Acoustics of the glass harmonica," J. Acoust. Soc. Amer., vol. 95, no. 2, pp. 1106-1111, 1994.

[33] S. Serafin, F. Avanzini, and D. Rocchesso, "Bowed string simulation using an elasto-plastic friction model," in Proc. Stockholm Music Acoustics Conf. (SMAC 2003), Stockholm, Sweden, Aug. 2003, pp. 95-98.

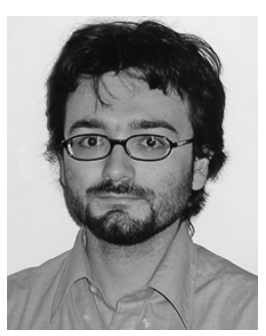

Federico Avanzini received the Laurea degree in physics from the University of Milano, Italy, in 1997, with a thesis on nonlinear dynamical systems and full marks. From November 1998 to November 2001, he pursued the Ph.D. degree in computer science at the University of Padova, Italy, with a research project on computational issues in physically based sound models.

Within his doctoral activities, he worked as a Visiting Researcher at the Laboratory of Acoustics and Audio Signal Processing, Helsinki University of Technology, Helsinki, Finland. He is now Assistant Professor, Department of Information Engineering, University of Padova. His research interests include sound processing and physically-based sound synthesis, audio rendering in multimodal human-computer interfaces, voice production, and articulatory modeling. 


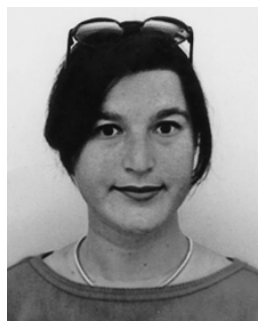

Stefania Serafin was born in Venice, Italy, where she received the Laurea degree (summa cum laude) in computer science from the University of Venice, Italy, in 1996. At the same time, she studied violin and music theory at the Music Conservatory B. Marcello of Venice. She received the Ph.D. degree from CCRMA, Stanford University, Stanford, CA. In 1997, she moved to Paris, France, where she worked in the Analysis-Synthesis Team at Ircam, researching physical models of bowed strings and studying for the Master's degree in acoustics, signal processing, and computer science applied to music.

Since then, she has been pursuing her research in labs such as CCRMA, Stanford University, Stanford, CA; Cambridge University, Cambridge, U.K.; $\mathrm{KTH}$, Stockholm, Sweden, and the University of Virginia, Charlottesville. She recently moved to Aalborg University Copenhagen, Denmark, where she is Assistant Professor of medialogy with a focus on musical applications.

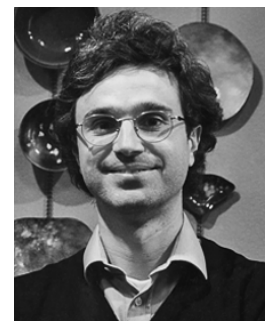

Davide Rocchesso received the Laurea degree in electronic engineering and the Ph.D. degree from the University of Padova, Padua, Italy, in 1992 and 1996, respectively. His Ph.D. research involved the design of structures and algorithms based on feedback delay networks for sound processing applications.

In 1994 and 1995, he was a Visiting Scholar at the Center for Computer Research in Music and Acoustics (CCRMA), Stanford University, Stanford, CA. Since 1991, he has been collaborating with the Center of Computational Sonology (CSC), University of Padova, as a Researcher and Live-Electronics Designer. Since 1998, he has been with the University of Verona, Verona, Italy, where he is now Associate Professor. At the Dipartimento di Informatica, University of Verona, he coordinated the project Sounding Object, funded by the European Commission within the framework of the Disappearing Computer initiative. His main interests are in audio signal processing, physical modeling, sound reverberation and spatialization, multimedia systems, and human-computer interaction. 